\title{
Obesity, Hypersomnolence, and Quality of Sleep: the Impact of Bariatric Surgery
}

\author{
Thisciane Ferreira Pinto ${ }^{1}$ • Pedro Felipe Carvalhedo de Bruin ${ }^{1}$. \\ Veralice Meireles Sales de Bruin ${ }^{1}$ - Paulo Marcos Lopes ${ }^{2}$ - Francisco Ney Lemos ${ }^{2}$
}

(C) Springer Science+Business Media New York 2017

\begin{abstract}
Purpose Obesity is commonly associated with poor sleep, excessive daytime sleepiness (EDS) and depressive mood but the impact of bariatric surgery on these conditions is incompletely understood. This study aimed to investigate the course of EDS and sleep quality in bariatric surgery patients in relation with changes in body weight and depressive symptoms.

Methods In patients consecutively submitted to bariatric surgery, baseline and postoperative sleep quality were evaluated by the Pittsburgh Sleep Quality Index (PSQI), excessive daytime sleepiness by the Epworth Sleepiness Scale (ESS), risk for OSA by the Berlin Questionnaire (BQ), and depressive symptoms by the Beck Depression Inventory-Short Form (BDI). Comorbidities were assessed by interview and chart review.

Results Sixty patients $(\mathrm{M} / \mathrm{F}=9 / 51)$ with a mean $( \pm \mathrm{SD})$ age of $34.7 \pm 9.2$ years and body mass index (BMI) of $46.04 \pm 7.52 \mathrm{~kg} / \mathrm{m}^{2}$ were studied. Bariatric surgery improved PSQI score $(6.4 \pm 3.8$ versus $4.1 \pm 2.8 ; p<0.001)$, ESS score $(8.1 \pm 4.7$ versus $6.0 \pm 3.3 ; p<0.001)$, BDI score $(9.8 \pm 7.0$ versus $4.7 \pm 4.6 ; p=0.001$ ), and risk for OSA (68.3 versus $5 \%$ ). Twelve of the 18 subjects with baseline EDS (ESS $\geq 10$ ) developed normal ESS score after surgery. In these subjects, significant postoperative improvement in depressive symptoms score was observed $(12.0 \pm 9.0$ versus $5.5 \pm 5.0$;
\end{abstract}

Pedro Felipe Carvalhedo de Bruin pedrobruin@gmail.com

1 Laboratory of Sleep and Biological Rhythms, Department of Medicine, Universidade Federal do Ceará, Rua Professor Costa Mendes, 1.608/4th floor, Fortaleza, CE 60430-140, Brazil

2 Hospital Geral Dr. César Cals, Fortaleza, Brazil $p=0.041$ ), in contrast to the remaining six cases with persistent EDS, who showed no significant change in these symptoms $(5.5 \pm 5.0$ versus $3.2 \pm 3.1 ; p=0.416)$.

Conclusion Bariatric surgery has a beneficial effect on sleep quality and EDS. Postoperative improvement in EDS can be related to a reduction in depressive symptoms.

Keywords Obesity · Disorders of excessive somnolence · Depression $\cdot$ Sleep

\section{Introduction/Purpose}

Obesity is a public health problem with a major negative impact on morbidity, mortality, and health-related quality of life on a global scale [1]. Most studies on the causes and treatment of obesity have so far focused on caloric ingestion and energy expenditure. More recently, the role of sleep in metabolic regulation has been increasingly recognized as well as the impact of insufficient and disrupted sleep on the development of the metabolic syndrome [2, 3]. Impaired sleep, in association with changes in lifestyle and socioeconomic environment, is increasingly common in modern society. It has been reported that one in five adults may be currently affected by sleep problems [4]. The rapid increase in the prevalence of overweight/obesity appears to have occurred in tandem with the gradual worsening of sleep inadequacy over the last three decades, as suggested by a recent analysis of a large dataset of non-institutionalized US adults [2, 5]. Poor quality sleep and short sleep duration have been shown to enhance positive energy balance and result in weight gain through several mechanisms, including effects on sleep metabolic rate, leptin and ghrelin concentrations, appetite, hypothalamic-pituitaryadrenal axis activity, gut-peptide concentrations, and substrate oxidation [6]. On the other hand, obese subjects are more 
likely to suffer from inadequate sleep [7, 8], thus pointing to a reciprocal relationship, whereby poor sleep leads to weight gain which may, in turn, induce more sleep impairment.

Excessive daytime sleepiness is common in obese subjects and has a negative impact on their safety and quality of life. Obstructive sleep apnea (OSA) and sleep loss are generally considered the main determinants of daytime sleepiness in obesity. However, despite the close association between OSA and obesity, the relation between severity of OSA, measured by the apnea-hypopnea index, and that of the excessive daytime sleepiness, as assessed by the Epworth Sleepiness Scale (ESS), is weak. In addition, sleepiness may persist in some cases despite an effective treatment for OSA [9, 10]. Other factors that have been suggested to play a role in the development of the excessive daytime sleepiness in obese patients include depression and metabolic disorders, such as insulin resistance and diabetes [11, 12].

Bariatric surgery is considered an effective treatment for severe obesity and may have a beneficial effect on its comorbidities, such as disturbed sleep and its daytime consequences [13]. However, factors associated with persistence of hypersomnolence after bariatric surgery have not been elucidated. The main purpose of this study was to investigate the longitudinal course of excessive daytime sleepiness and sleep impairment in patients undergoing bariatric surgery in relation with changes in mood and weight loss.

\section{Methods}

Patients of both genders aged 18 years or older, with body mass index (BMI) $\geq 40$ or $\geq 35$ and at least one comorbidity related to obesity condition, were approached to take part in the study, on the occasion of their first visit to the Obesity Surgery Outpatient Clinic at "Hospital Geral Dr. César Cals," in Fortaleza, Brazil. Individuals with heart, kidney or liver failure, neoplasms, and women who were pregnant or breastfeeding were excluded from the study. The protocol was approved by the local Research Ethics Committee, and all participants provided the signed written informed consent before the procedures (approval number-442/2010).

Sociodemographic and clinical data, including presence of diabetes mellitus and systemic arterial hypertension, were obtained by interview and chart review. Body mass index was calculated as the ratio between weight $(\mathrm{kg})$ and squared height $\left(\mathrm{m}^{2}\right)$. Quality of sleep was evaluated by the Pittsburgh Sleep Quality Index (PSQI), a self-report questionnaire that assesses sleep quality over a 1-month time interval. It consists of 19 items generating seven component scores: subjective sleep quality, sleep latency, sleep duration, sleep efficiency, daytime dysfunction, use of medications to sleep, and presence of sleep disorders. Individuals with a total PSQI score greater than five were considered poor sleepers. A Portuguese-language version of the PSQI adapted to the Brazilian population was used in this study [14]. Daytime sleepiness was assessed using the ESS, a questionnaire which includes eight items regarding the expectation of dozing in eight hypothetical situations. A score of 10 or above was considered indicative of excessive daytime sleepiness. A Portuguese-language version of the ESS previously adapted to the Brazilian population was used in this study [15]. Risk for OSA was assessed by a Portugueselanguage version of the Berlin Questionnaire (BQ) that had been previously translated and adapted to the Brazilian population [16]. Presence and severity of the depressive symptoms were investigated by the Beck Depression Inventory-Short Form (BDI) and symptoms were classified as mild ( $>4$ to 7 points), moderate ( $>7$ to 15 points), or severe ( $>15$ points). A Portuguese-language version of the BDI, adapted to the Brazilian population, was used in this study [17].

All patients were routinely evaluated by a multidisciplinary team and a laparoscopic Roux-en-Y gastric bypass was performed in all cases. Measurements and questionnaires were repeated postoperatively, at least $\operatorname{six}($ mean $\pm \mathrm{SD}=16.1 \pm 6.0$ ) months after surgery, for comparison.

\section{Data Analysis}

Values are presented as mean \pm SD or frequency. Participants were divided into two groups according to the presence (ESS $\geq 10$ ) or absence of preoperative excessive daytime sleepiness for analysis. Data were examined for normality using the Kolmogorov-Smirnov test. For normally distributed variables with homogeneity of variance, between-group comparisons were performed using Student's unpaired $t$ test and within-group comparisons using the paired $t$ test. The Wilcoxon test was used for comparisons of before and after bariatric surgery of those variables that did not meet the requirements for homogeneity of variances. Categorical variables were analyzed by the chi-square or McNemar test. Pearson's correlation coefficients were calculated to evaluate the relation between BQ, BMI, and ESS scores. Analyses were conducted using the Statistical Package for the Social Sciences (SPSS) for Windows, version 20.0 (SPSS Inc., Chicago, IL, USA). Differences were considered to be significant at $p$ value $<0.05$.

\section{Results}

Sixty patients consecutively submitted to bariatric surgery, aged between 21 and 56 (mean $\pm \mathrm{SD}=34.7 \pm 9.2$ ) years, with a BMI ranging from 35.0 to $72.4 \mathrm{~kg} / \mathrm{m}^{2}$, were included in the study. General characteristics and results of the basal and follow-up PSQI, ESS, and BQ scores are summarized in Table 1. Risk of OSA, as measured by the BQ, was positively 
Table 1 Sleep quality, excessive daytime sleepiness, depressive symptoms, comorbidities, and risk of obstructive sleep apnea in 60 obese patients at baseline and after bariatric surgery

\begin{tabular}{lccr}
\hline Variables & Baseline & After & $p$ value \\
\hline BMI $\left(\mathrm{kg} / \mathrm{m}^{2}\right)$ & $46.0 \pm 7.5$ & $30.3 \pm 4.3$ & $<0.001^{\mathrm{a}}$ \\
Diabetes mellitus & $10(16 \%)$ & $2(3.3 \%)$ & $0.008^{\mathrm{c}}$ \\
Hypertension & $25(41.7 \%)$ & $13(21.7 \%)$ & $0.002^{\mathrm{c}}$ \\
PSQI score & $6.4 \pm 3.8$ & $4.1 \pm 2.8$ & $<0.001^{\mathrm{b}}$ \\
Impaired sleep & $31(51.7 \%)$ & $17(28.3 \%)$ & $0.009^{\mathrm{c}}$ \\
ESS score & $8.1 \pm 4.7$ & $6.0 \pm 3.3$ & $<0.001^{\mathrm{b}}$ \\
EDS & $18(30 \%)$ & $6(10 \%)$ & $<0.001^{\mathrm{c}}$ \\
BDI score & $9.7 \pm 7.0$ & $4.7 \pm 4.6$ & $<0.001^{\mathrm{b}}$ \\
Moderate/severe depressive symptoms & $33(55 \%)$ & $14(23.3 \%)$ & $0.001^{\mathrm{c}}$ \\
BQ & $41(68.3 \%)$ & $3(5 \%)$ & $<0.001^{\mathrm{c}}$ \\
\hline
\end{tabular}

Data are expressed as means \pm standard deviation

$B D I$ Beck Depression Inventory-Short Form, $B M I$ body mass index, $B Q$ Berlin Questionnaire, $E D S$ excessive daytime sleepiness, ESE Epworth Sleepiness Scale, PSQI Pittsburgh Sleep Quality Index

${ }^{\text {a }}$ Student's paired $t$ test

${ }^{\mathrm{b}}$ Wilcoxon test

${ }^{\mathrm{c}}$ McNemar test

correlated with the BMI $(R=0.34 ; p=0.007)$ and presence of excessive daytime sleepiness $(R=0.30 ; p=0.017)$.

After the bariatric surgery, there was an improvement in the following components of the PSQI: quality of sleep $(p=0.041)$, latency of sleep $(p<0.001)$, disturbance of sleep $(p<0.001)$, and tendency for improvement in daytime dysfunctions $(p=0.05)$.

Twelve of the 18 subjects with baseline excessive daytime sleepiness $(E S S \geq 10)$ developed a normal ESS score after surgery. In these subjects, significant postoperative improvement in depressive symptoms score was observed (12.0 \pm 9.0 versus $5.5 \pm 5.0 ; p=0.041$ ), in contrast to the remaining six cases with persistent excessive daytime sleepiness, who showed no significant change in these symptoms $(5.5 \pm 5.0$ versus $3.2 \pm 3.1 ; p=0.416$ ). In the present study, persistent daytime somnolence was not associated with the degree of weight loss or the magnitude of improvement in subjective sleep quality after bariatric surgery.

\section{Discussion}

This study confirms the beneficial effect of bariatric surgery on subjective sleep quality and hypersomnolence and suggests that persistence of excessive daytime sleepiness after surgery may be related to a lack of improvement in depressive symptoms.

Sleep quality improvement after bariatric surgery has been previously reported $[8,18,19]$. Obesity is the main risk factor for OSA, a major cause of disturbed sleep and excessive daytime sleepiness in this patient population, and previous studies confirm the effectiveness of obesity surgery for the control the OSA $[19,20]$. More than half of the participants in the present study were identified as being at risk for OSA at baseline, as assessed by the Berlin Questionnaire (BQ), a figure that decreased dramatically after the surgical intervention (5.0\%). However, it should be kept in mind that overnight polysomnography is the gold standard for the diagnosis of OSA and although clinical screening tools, such as the BQ, have been widely used for this purpose, the findings regarding their diagnostic accuracy are controversial. A meta-analysis of 26 studies has suggested that among other screening tools, the BQ presents the highest diagnostic odds ratio [21].

The majority of the subjects in the present study showed an improvement in excessive daytime sleepiness after surgery, in agreement with previous investigations [22-24]. It has been suggested that the decrease in hypersomnolence after bariatric surgery may be related to an improvement in nocturnal sleep, secondary to the reduction in OSA, as mentioned above, although other factors, such as a reduction of metabolic and inflammatory abnormalities, could also play a role $[9,25$, 26]. In a previous study of 56 obese patients with high-level preoperative daytime somnolence, normalization of excessive sleepiness was achieved only 1 month after the surgical procedure [27]. It is worth emphasizing that about $10.0 \%$ of the participants of the present study were found to remain with excessive daytime sleepiness at the postoperative evaluation. The causes of persistent hypersomnolence after successful obesity surgery have not been adequately clarified $[9,10]$. In this study, we were unable to find a correlation between persistence of excessive daytime sleepiness and the magnitude of sleep quality improvement or the degree of weight loss after the surgical procedure.

An overall improvement in depressive symptoms after bariatric surgery was observed in this study. Depressive symptomatology is commonly found in obese subjects and this relation may be bi-directional [28]. It has been suggested that obesity 
can lead to depressive mood for several reasons, including physical inactivity, poorer quality of life, and social prejudice [29-31]. On the other hand, observations that depressed individuals may gain weight because of the use of antidepressants, sedentarism, and poor sleep quality [32,33] seem to support a reverse sequence of events. Finally, the co-occurrence of these two conditions must also be considered in view of common genetic and lifestyle factors [32, 34]. Significant improvement in depressive symptoms has been previously reported following bariatric surgery [35], and greater weight loss has been correlated to higher rates of depression resolution in these individuals [36]. The nature of the postoperative improvement of depressive symptoms is probably multifactorial and may be related to psychosocial factors, including gains in self-esteem, body satisfaction, and weight-related stigmatization, as well as biological factors, such as reduction in insulin resistance, deactivation of pro-inflammatory states and normalization of the hypothalamic-pituitary-adrenal axis $[37,38]$. A relevant finding of the present study is the concomitant improvement in depressive symptoms among subjects who experienced normalization of daytime sleepiness scores, in contrast to the absence of a change in depressive symptoms among those with persistent daytime somnolence. A relationship between obesity, depression, and excessive daytime sleepiness has been previously described in community cohort studies [39] as well as in obese subjects [40]. Dixon and coworkers, in 1055 consecutive patients presenting for obesity surgery, observed that daytime somnolence, as measured by the ESS, was not related to the presence or severity of OSA diagnosed by overnight polysomnography in a subgroup of subjects. The largest contributors to variance of ESS scores in their sample were symptoms of depression, sleep problems reported by the patient, and poor quality of life [11]. Prospective studies evaluating the associations among depressive symptoms, sleep quality, and excessive daytime sleepiness following bariatric surgery are scarce. However, Fernandez-Mendoza and coworkers, in a population-based study of 1395 subjects followed up after 7.5 years, reported that weight loss was associated with remission of excessive daytime sleepiness. Interestingly, those investigators also found that the presence of sleep disturbances could predict the incidence of daytime somnolence in individuals with depression [25].

Frequency of diabetes mellitus and systemic arterial hypertension decreased significantly after surgery among the participants of this study, thus confirming previous reports on the positive effect of obesity surgery on these conditions [13, 41]. As diabetes mellitus has been found to be a predictor of excessive daytime sleepiness, regardless of obesity [25], this reduction may also have contributed to the overall improvement in daytime somnolence evidenced in the present results.

In this study, daytime sleepiness was not objectively measured. The multiple sleep latency test (MSLT), which quantifies the ability to fall asleep on multiple repeated nap opportunities, is generally regarded as the gold standard objective measure of excessive sleepiness [42]. However, there is evidence that the MSLT has limitations for the assessment of sleepiness in subjects with depressive symptoms [43, 44]. In this study, subjective sleepiness was assessed by the ESS, the most commonly used measure of sleepiness in sleep research and clinical settings [45]. Recently, Plante and coworkers examined the associations of depression with habitual sleep duration, subjective daytime sleepiness, and objective sleep propensity in a nonclinical adult population. Those investigators found that subjective daytime sleepiness and increased habitual sleep duration were associated with increased odds of depression. Paradoxically, increased sleep propensity on the MSLT was associated with reduced likelihood of depression, underscoring the limitations of the MSLT as a measure of sleepiness in the context of mood disorders [46].

In conclusion, bariatric surgery leads to an overall improvement in subjective sleep quality and daytime somnolence. Excessive daytime sleepiness may persist after surgery in a small but significant proportion of individuals. Postoperative improvement in hypersomnolence can be related to an improvement in depressive symptoms. Further studies are necessary to fully clarify the mechanisms involved in the relation between obesity, hypersomnolence, and depression.

Acknowledgements Thisciane F. Pinto was the recipient of a scholarship from Coordenação de Aperfeiçoamento de Pessoal de Nivel Superior (CAPES), Brazil. The authors would like to thank Mr. Antonio Brazil Viana Jr. for his technical assistance.

\section{Compliance with Ethical Standards}

Conflict of Interest The authors declare that they have no conflict of interest.

Informed Consent Informed consent was obtained from all individual participants included in the study.

Ethical Approval All procedures performed in this study involving human participants were in accordance with the ethical standards of the institutional and/or national research committee and with the 1964 Helsinki Declaration and its later amendments or comparable ethical standards.

\section{References}

1. Wang YC, McPherson K, Marsh T, et al. Health and economic burden of the projected obesity trends in the USA and the UK. Lancet. 2011;378:815-25.

2. Lauderdale DS, Knutson KL, Rathouz PJ, et al. Cross-sectional and longitudinal associations between objectively measured sleep duration and body mass index: the CARDIA sleep study. Am J Epidemiol. 2009;170:805-13. 
3. Taheri S. The link between short sleep duration and obesity: we should recommend more sleep to prevent obesity. Arch Dis Child. 2006;91:881-4.

4. Krueger PM, Friedman EM. Sleep duration in the United States: a cross-sectional population-based study. Am J Epidemiol. 2009;169: 1052-63.

5. Jean-Louis G, Williams NJ, Sarpong D, et al. Associations between inadequate sleep and obesity in the US adult population: analysis of the national health interview survey (1977-2009). BMC Public Health. 2014;14:290.

6. Gonnissen HK, Hulshof T, Westerterp-Plantenga MS. Chronobiology, endocrinology, and energy- and food-reward homeostasis. Obes Rev. 2013;14:405-16.

7. Bidulescu A, Din-Dzietham R, Coverson DL, et al. Interaction of sleep quality and psychosocial stress on obesity in African Americans: the cardiovascular health epidemiology study (CHES). BMC Public Health. 2010;10:581.

8. Toor P, Kim K, Buffington CK. Sleep quality and duration before and after bariatric surgery. Obes Surg. 2012;22:890-5.

9. Panossian LA, Veasey SC. Daytime sleepiness in obesity: mechanisms beyond obstructive sleep apnea-a review. Sleep. 2012;35:605-15.

10. Pedrosa RP, Lorenzi-Filho G, Drager LF. Síndrome da apnéia obstrutiva do sono e doença cardiovascular. Revista de Medicina. 2013;87:121-7.

11. Dixon JB, Dixon ME, Anderson ML, et al. Daytime sleepiness in the obese: not as simple as obstructive sleep apnea. Obesity. 2007; 15:2504-11.

12. Vgontzas AN, Bixler EO, Chrousos GP, et al. Obesity and sleep disturbances: meaningful sub-typing of obesity. Arch Physiol Biochem. 2008;114:224-36.

13. Puzziferri N, Roshek TB, Mayo HG, et al. Long-term follow-up after bariatric surgery: a systematic review. JAMA. 2014;312:934-42.

14. Bertolazi AN, Fagondes SC, Hoff LS, et al. Validation of the Brazilian Portuguese version of the Pittsburgh Sleep Quality Index. Sleep Med. 2011;12:70-5.

15. Bertolazi AN, Fagondes SC, Hoff LS, et al. Portuguese-language version of the Epworth Sleepiness Scale: validation for use in Brazil. J Bras Pneumol. 2009;35:877-83.

16. Vaz A, Drummond M, Caetano Mota P, et al. Tradução do Questionário de Berlim para língua Portuguesa e sua aplicação na identificação da SAOS numa consulta de patologia respiratória do sono. Revista Portuguesa de Pneumología. 2011;17:59-65.

17. Furlanetto LM, Mendlowicz MV, Romildo BJ. The validity of the Beck Depression Inventory-Short Form as a screening and diagnostic instrument for moderate and severe depression in medical inpatients. J Affect Disord. 2005;86:87-91.

18. Dilektasli E, Dilektasli AG. Laparoscopic sleeve gastrectomy improves excessive daytime sleepiness and sleep quality 6 months following surgery: a prospective cohort study. Adv Ther. 2016;33:774-85.

19. Xie H, Doherty L, O'Boyle C. The positive impact of bariatric surgery on sleep. Ir Med J. 2016;109:328-30.

20. Sarkhosh K, Switzer NJ, El-Hadi M, et al. The impact of bariatric surgery on obstructive sleep apnea: a systematic review. Obes Surg. 2013;23:414.

21. Ramachandran SK, Josephs LA. A meta-analysis of clinical screening tests for obstructive sleep apnea. Anesthesiology. 2009;110: 928-39.

22. Dixon J, Schachter L, O'Brien P. Polysomnography before and after weight loss in obese patients with severe sleep apnea. Int J Obes. 2005;29:1048-54.

23. Holty JE, Parimi N, Ballesteros M, et al. Does surgically induced weight loss improve 305 daytime sleepiness? Obes Surg. 2011;21: 1535-45.

24. Fritscher LG, Canani S, Mottin CC, et al. Bariatric surgery in the treatment of obstructive sleep apnea in morbidly obese patients. Respiration. 2007;74:647-52.
25. Fernandez-Mendoza J, Vgontzas AN, Kritikou I, et al. Natural history of excessive daytime sleepiness: role of obesity, weight loss, depression, and sleep propensity. Sleep. 2015;38:351-60.

26. Kapur VK, Baldwin CM, Resnick HE, et al. Sleepiness in patients with moderate to severe sleep-disordered breathing. Sleep. 2005;28:472-7.

27. Varela JE, Hinojosa MW, Nguyen NT. Resolution of obstructive sleep apnea after laparoscopic gastric bypass. Obes Surg. 2007;17: 1279-82.

28. Mannan M, Mamun A, Doi S, et al. Is there a bi-directional relationship between depression and obesity among adult men and women? Systematic review and bias-adjusted meta-analysis. Asian J Psychiatr. 2016;21:51-66.

29. Heo M, Pietrobelli A, Wang D, et al. Obesity and functional impairment: influence of comorbidity, joint pain, and mental health. Obesity. 2010;18(10):2030-8.

30. Fontaine KR, Barofsky I. Obesity and health-related quality of life. Obes Rev. 2001;2(3):173-182.

31. Puhl RM, Brownell KD. Confronting and coping with weight stigma: an investigation of overweight and obese adults. Obesity. 2006;14(10):1802-15.

32. Stunkard AJ, Faith MS, Allison KC. Depression and obesity. Biol Psychiatry. 2003;54(3):330-7.

33. Riemann D, Berger M, Voderholzer U. Sleep and depression - results from psychobiological studies: an overview. Biol Psychol. 2001;57(103):67.

34. Anton SD, Newton Jr RL, Sothern M, et al. Association of depression with body mass index, sedentary behavior, and maladaptive eating attitudes and behaviors in 11 to 13 -year old children. Eat Weight Disord. 2006;11(3):e102-8.

35. Switzer NJ et al. The impact of bariatric surgery on depression: a review. Current Cardiovascular Risk Reports. 2016;10(3):1-5.

36. Burgmer R, Legenbauer T, Muller A, et al. Psychological outcome 4 years after restrictive bariatric surgery. Obes Surg. 2014;24(10): $1670-8$.

37. Dawes AJ, Maggard-Gibbons M, Maher AR, et al. Mental health conditions among patients seeking and undergoing bariatric surgery: a meta-analysis. JAMA. 2016;315:150-63.

38. de Zwaan M, Enderle J, Wagner S, et al. Anxiety and depression in bariatric surgery patients: a prospective, follow-up study using structured clinical interviews. J Affect Disord. 2011;133(1-2):61-8.

39. Kim H, Young T, Kim H, et al. Subjective daytime sleepiness: dimensions and correlates in the general population. Sleep. 2005;28(5):625-34.

40. Araghi $\mathrm{MH}$, Jagielski A, Neira I, et al. The complex associations among sleep quality, anxiety depression, and quality of life in patients with extreme obesity. Sleep. 2013;36(12):1859-65.

41. Courcoulas AP, Christian NJ, Belle SH, et al. Weight change and health outcomes at 3 years after bariatric surgery among individuals with severe obesity. JAMA. 2013;310:2416-25.

42. Littner MR, Kushida C, Wise M, et al. Practice parameters for clinical use of the multiple sleep latency test and the maintenance of wakefulness test. Sleep. 2005;28:113-21.

43. Billiard M, Dolenc L, Aldaz C, et al. Hypersomnia associated with mood disorders: a new perspective. J Psychosom Res. 1994;38:41-7.

44. Nofzinger EA, Thase ME, Reynolds 3rd CF, et al. Hypersomnia in bipolar depression: a comparison with narcolepsy using the multiple sleep latency test. Am J Psychiatry. 1991;148:1177-81.

45. Kendzerska TB, Smith PM, Brignardello-Petersen R, et al. Evaluation of the measurement properties of the Epworth Sleepiness Scale: a systematic review. Sleep Med Rev. 2014;18: 321-31.

46. Plante DT, Finn LA, Hagen EW, et al. Subjective and objective measures of hypersomnolence demonstrate divergent associations with depression among participants in the Wisconsin Sleep Cohort study. J Clin Sleep Med. 2016;12:571-8. 